\title{
Recovery of a Patient from a Seemingly Vegetative State
}

\section{Nevine M. El Nahas}

Ain Shams University, Cairo, Egypt

\begin{abstract}
Vegetative state (VS) is characterized by complete absence of behavioural evidence for self or environmental awareness. This is a case report of a traumatic brain injury (TBI) patient who was referred as a vegetative state. He was given medical, cognitive and physical therapy and recovered consciousness after 6 months. We thus concluded that patients with such a diagnosis should receive best medical, physical and cognitive care as some of them could show good recovery.
\end{abstract}

Keywords: Vegetative state; Minimally conscious state; Rehabilitation

\section{Introduction}

Vegetative state is defined as a state of wakefulness without awareness in which there is preserved capacity for spontaneous or stimulus-induced arousal, evidenced by sleep-wake cycles and a range of reflexive and spontaneous behaviours. B.M., a male patient 26 years old, had a car accident two months prior to admission to our Neurorehabilitation Centre in Cairo. It resulted in traumatic brain injury with all radiological investigations excluding any spinal cord trauma. He presented to us with a picture suggestive of vegetative state, glascow coma scale 6 . He had a normal sleep waking rhythm, yet could not respond to verbal stimuli even by eye movements. He didn't show following eye movements or any sign of attention to surroundings. The patient had severe involuntary movements mainly in the lower limbs, a mixture of proximal ballistic movements and distal dystonia of both feet. Active power could not be assessed; however he showed spontaneous movements of right upper and lower extremities, and flexion to pain on the left side.

MRI brain and EEG showed no abnormalities [1].

\section{Procedures and Plan of Management}

Cognitive enhancement was performed by our cognitive therapist, mainly by intensive auditory, visual and tactile stimuli.

Control of involuntary movements by anticholinergics and clonazepam. Repetitive transcranial magnetic stimulation rTMS 1 $\mathrm{hz}$ for 15 minutes on the dominant motor area M1 was given daily to inhibit movements. After about 2 weeks, involuntary movements started to subside, and the patient began to show signs of attention. He showed following eye movements to visual and auditory stimuli, emotional response on seeing relatives and non-comprehensible sounds. At that point we started physical rehabilitation by tilt Table training, strengthening exercises to both lower limbs and training to stand with bilateral support.

The patient was discharged for follow up. On reevaluation 6 month later, the patient was fully conscious, oriented for time, place and persons. He even was able to recollect personnel in our centre. He could talk with slurred speech and walk with bilateral support. He had a crouch gait due to flexion deformities in both knees.

He seemed to have some behavioural changes being slightly disinhibited and jocular. He was referred to orthopedic surgeon for tendon lengthening of knee flexors to be followed by gait rehabilitation [2].

\section{Discussion}

The patient presented here fulfilled the definition of VS rather than Minimal Conscious State (MCS), the later being wakefulness with minimal awareness.

However, it seems that differentiation between both conditions is difficult on clinical grounds.

He regained full consciousness 10 month after trauma. He started to show awareness in our rehabilitation center. On discharge he could respond to orders, recognize his mother and stand with bilateral support. He seems to have continued improvement over the six month post-discharge to the extent of full awareness and orientation for persons, time and places, appropriate verbal and motor response. $\mathrm{He}$ had residual contractures and disinhibited behaviour.

Recovery was initially unexpected and we cannot tell which procedure aided in such response.

Other studies reported similar results. Estraneo et al. 2013 reported 13 patients who showed variable degrees of recovery from VS 1 year after TBI, 6 of whom became conscious yet had cognitive impairments not impeding their interaction with relatives. Another series of 9 patients with severe TBI and initial glascow coma scale scores 3-6, reported full functional recovery of all nine patients by 8 to 15 months. They received outpatient rehabilitation from 3 to 9 months, the details of which are not specified $[3,4]$.

\section{Limitations}

This is a report of recovery of only one patient that cannot be generalized to other patients with a similar condition. We cannot identify the actual procedure that induced recovery whether intensive stimulation, rTMS, medications or all of them.

\section{Conclusion}

This patient initially seemed to be in a vegetative state, suggestive of

*Corresponding author: Nevine M. El Nahas, Department of Neurology, Ain Shams University, Cairo, Egypt, Tel: 00201227910517; E-mail: nevineelnahas30@yahoo.com Received October 27, 2014; Accepted January 20, 2015; Published January 22 2015

Citation: Nahas NME (2015) Recovery of a Patient from a Seemingly Vegetative State . J Trauma Treat 4: 233. doi:10.4172/2167-1222.1000233

Copyright: (c) 2015 Nahas NME. This is an open-access article distributed under the terms of the Creative Commons Attribution License, which permits unrestricted use, distribution, and reproduction in any medium, provided the original author and source are credited. 
bad prognosis. However, cognitive and sensory stimuli and control of involuntary movements revealed that he was in a minimally conscious state that responded to proper, targeted medical care. Improvement continued to progress over six month after discharge from our facility.

\section{Recommendation}

Every TBI patient should be given best rehabilitation care including cognitive and physical therapy even if he seemed to be in a vegetative state. It is still difficult, for the time being, to distinguish between vegetative state and minimally conscious state. So, each brain injured patient should be given the benefit of doubt and receive the best medical care. The role of rTMS has yet to be studied.

\section{References}

1. Royal College of Physicians. Prolonged disorders of consciousness (2013) National clinical guidelines. Report of working party.

2. Giacino JT, Kalmar K (2005) Diagnostic and prognostic guidelines for the vegetative and minimally conscious states. Neuropsychol Rehabil 15: 166-174.

3. Estraneo A, Moretta P, Loreto V, Santoro L, Trojano L (2014) Clinical and neuropsychological long term outcomes after late recovery of responsiveness: a case series. Arch Phys Med and Rehabil 95: 71

4. Kushner DS, Johnson-Greene D (2014) Changes in cognition and continence as predictors of rehabilitation outcomes in individuals with severe traumatic brain injury. J Rehabil Res Dev 51: 1057-1068. 